\title{
Frailty and Disability in Diabetes
}

\author{
Sol-Ji Yoon ${ }^{1}$, Kwang-il Kim ${ }^{2,3}$ \\ ${ }^{1}$ Departement of Internal Medicine, Kangwon National University Hospital, Chuncheon, Korea \\ ${ }^{2}$ Department of Internal Medicine, Seoul National University Bundang Hospital, Seongnam, Korea \\ ${ }^{3}$ Department of Internal Medicine, Seoul National University College of Medicine, Seoul, Korea
}

\author{
Corresponding Author: \\ Kwang-il Kim, MD, PhD \\ Department of Internal Medicine, \\ Seoul National University Bundang \\ Hospital, 82 Gumi-ro 173beon-gil, \\ Bundang-gu, Seongnam 13620, Korea \\ E-mail: kikim907@snu.ac.kr \\ ORCID:
}

https://orcid.org/0000-0002-6658-047X

Received: October 25, 2019

Revised: November 29, 2019

Accepted: December 1, 2019
Diabetes is an important health problem with the population aging. Previously, it is well established that diabetes is associated with microvascular and macrovascular complications, but recently, several data suggest that diabetes is accompanied with frailty as well as disability among the older adults. Considering the clinical significance of frailty and disability, it is important to understand the pathway from diabetes to frailty and/or disability. Additionally, it is strongly recommended to find a new therapeutic intervention are required to meet the increasing demand of managing older diabetic patients with the population aging.

Key Words: Frail elderly, Diabetes mellitus, Disability

\section{INTRODUCTION}

Diabetes is a serious health condition for older adults that has an increasing clinical significance in aged societies. More than 25\% of Korean adults over 60 years of age have diabetes mellitus, and approximately $50 \%$ of older adults have impaired fasting glucose levels. ${ }^{1,2)}$ Diabetes is associated with complications including cardiovascular disease, retinopathy, renal failure, and peripheral vascular disease. The occurrence of microvascular or macrovascular diabetic complications increases significantly after 10 years of disease duration. These complications are related to functional decline, disability, and loss of quality of life. ${ }^{3,4)}$ Among older adults with diabetes, geriatric syndrome, especially frailty, is emerging as a third category of complications in addition to the traditional microvascular or macrovascular diseases leading to considerable disability. Understanding the pathway from diabetes to frailty and/or disability is important for establishing early intervention and prevention strategies. This review discusses the relationship between frailty, disability, and diabetes among older adults.

\section{FRAILTY AND DISABILITY IN OLDER DIABETIC PATIENTS}

In recent years, frailty has emerged as an independent predictor associated with increased risks of adverse outcomes among older adults, irrespective of their associated comorbidities. Frailty is a state of decreased reserve and resistance to stressors resulting from cumulative declines across multiple physiologic systems with aging, which leads to vulnerability to adverse outcomes such as death, hospital admission, permanent institutionalization, falls, and additional disability. ${ }^{5)}$ While disability is hard to reverse, frailty is a dynamic process with a wide range of interventions to reduce vulnerability. Fried et al. ${ }^{6}$ established a framework of the frailty phenotype in which three or more of the following criteria were present: unintentional weight loss, self-reported exhaustion, muscle weakness, slow walking speed, and low physical disability. The presence of one or two phenotype criteria describes a pre-frail state while the absence of any positive criteria describes a robust state. Another definition of frailty is the frailty index proposed by Rockwood and colleagues. In this concept, Frailty is recognized as an accumulation of deficit during aging (symptoms, diseases, condi- 
tions, and disabilities). ${ }^{7)}$ Studies have showed that Frailty is a dynamic process and also potentially reversible through interventions in carefully chosen, evidence-based circumstances. ${ }^{8)}$

Several studies have shown that diabetic patients are more likely to be frail than non-diabetic older adults. ${ }^{9-12)}$ From the German ESTHER cohort study and Whitehall II Prospective Study, the prevalence of frailty in adults older than 65 years was 3- to 5-fold higher in patients with diabetes than that in the general population. $^{13,14)}$ Data from the National Health and Nutrition Examination Survey (NHANES) and Cardiovascular Heart Study (CHS) indicate that frailty and pre-frailty are present in $25 \%$ and $18.2 \%$ of individuals with diabetes, respectively, compared to an overall prevalence of $6.9 \%$ among those aged $\geq 65$ years. The Beijing Longitudinal Study of Aging II, demonstrated the highest prevalence of frailty among individuals with diabetes (19.3\%), compared to $11.4 \%$ and $11.9 \%$ in pre-diabetic and non-diabetic individuals, respectively. ${ }^{15)}$

The pathophysiology of diabetes is closely associated with frailty. Long-duration diabetes increases the loss of skeletal muscle mass and function, which lead to reduced mobility and gait speed. ${ }^{16)}$ Insulin resistance can aggravate lower limb dysfunction, further worsening the increase in intramyocellular fatty-acid metabolites caused by reduced mitochondrial activity that accompanies muscle aging. ${ }^{17)}$ This is likely due to the increased risk of sarcopenia, which is linked to frailty ${ }^{18)}$ Hyperglycemia is associated with increased insulin resistance, chronic inflammation, oxidative stress, and mitochondrial dysfunction, all of which have deleterious effects on skeletal muscle mass and function, leading to sarcopenia. ${ }^{19)}$

Because frailty might be caused by the accumulation of subclinical damage in multiple organ systems, diabetic complications may also lead to frailty. A Japanese cross-sectional study of 9,695 participants showed a significantly increased risk of frailty among individuals with a history of diabetes and lower kidney function (odds ratio $=2.76,95 \%$ confidence interval $[\mathrm{CI}], 1.21-8.24){ }^{20)}$

Among diabetic complications, autonomic neuropathy can cause orthostatic hypotension, arrhythmia, syncope, diarrhea, and bladder dysfunction. It may also result in the absence of typical hypoglycemic symptoms such as sweating, tachycardia, and tremors. It is also related to adverse events such as falls, malnutrition, and urinary incontinence and eventually leads to frailty.

Frailty is also associated with insulin resistance in the post-absorptive state of glucose metabolism in the presence of increased abdominal fat. A Canadian study showed lower insulin sensitivity in the frail-obese group than in the frail-lean group but observed no significant differences among the healthy, non-obese and frailobese or frail-lean groups. ${ }^{21)}$

Frailty is a major factor associated with an increased risk of death and disability in older adults with diabetes. Among 1,825 participants aged $\geq 65$ years in the Toledo Study of Healthy Aging (TSHA) cohort study, individuals with diabetes died more frequently than those without diabetes (hazard ratio $=1.36$; $95 \% \mathrm{CI}$, $1.06-1.75 ; \mathrm{p}=0.002$ ), showing a poorer functional status at baseline. The hazard ratios for death were 1.51 (95\% CI, 1.28-1.77) and 1.83 (95\% CI, 1.49-2.26) for each 10-point increase in Frailty Trait Score and Frailty Index, respectively. ${ }^{22)}$ An observational study of clinic patients with diabetes mellitus aged 50-90 years reported that participants with frailty were more likely to have new activities of daily living disability at the 6-month follow-up. ${ }^{23)}$

Accordingly, previous evidence confirmed the close relationship between diabetes and frailty and the adverse effect of frailty on clinical outcomes among older patients with diabetes. Older adults with diabetes and frailty may experience multiple medical conditions and be trapped in vicious cycles, which lead to further functional decline. Thus, preventive approaches in older patients with frailty and diabetes are important for maintaining their healthy lifestyles.

\section{COMMON PROBLEMS AMONG DIABETIC PATIENTS WITH FRAILTY OR DISABILITY}

\section{Hypoglycemia}

Older adults with frailty have an increased risk of hypoglycemia as most of these individuals have problems with loss of appetite and weight loss. Recurrent hypoglycemia is common in older patients with diabetes but is less likely to be recognized and, thus, is under-reported by patients and healthcare professionals. The lack of recognition is mainly due to the predominance of neurological symptoms (dizziness or visual disturbance) rather than autonomic symptoms (palpitation, sweating, anxiety, and nausea) associated with hypoglycemia among older adults as the autonomic symptoms are decreased and counter-regulatory responses to hypoglycemia are reduced in older people. ${ }^{24)}$ Severe hypoglycemia often leads to hospitalization in older adults, which could lead to the deterioration of a patient's general condition and, eventually, frailty and disability. Repeated hypoglycemia is likely to result in frailty, disability, and poor health outcomes. ${ }^{25)}$

\section{Falls}

Falls are a common problem in older adults with frailty. A Japanese study showed that lower walking speed, one of the manifestations of frailty, was associated with falls in patients with type 2 diabetes. The risk factors for falls among patients with diabetes include polypharmacy, muscle weakness, previous stroke, motor and sensory neuropathy, poor glycemic control, hypoglycemia, insulin use, 
cognitive dysfunction, orthostatic hypotension, and visual impairment. $^{26,27)}$ Fall prevention in patients with diabetes and frailty requires a multifactorial approach and there is strong evidence of a benefit in older adults. Gait, balance, and strength training might reduce the risk of falls in people with diabetes.

\section{Cognitive Dysfunction}

Frailty, and cognitive impairment, are prevalent among patients with cardiometabolic disease. ${ }^{28)}$ Moreover, diabetes is a risk factor for the development of vascular as well as neurodegenerative dementia. ${ }^{29)}$ Cognitive dysfunction significantly impacts the risk of both hypoglycemia and hyperglycemia in patients with diabetes. In particular, there is a bidirectional relationship between dementia and hypoglycemia; in other words, cognitive dysfunction is a risk factor for the development of hypoglycemia and hypoglycemia increases the risk of cognitive impairment in patients with diabetes. ${ }^{30)}$ Therefore, the prevention of hypoglycemia should always be considered a priority in the treatment of older patients with diabetes and frailty.

\section{Sarcopenia}

Patients who have diabetes mellitus, particularly when associated with renal failure, show an accelerated loss of muscle function. ${ }^{31,32)}$ Sarcopenia is an age-related decline in muscle function and mass. ${ }^{33)}$ Sarcopenia is associated with increased disability and mortality ${ }^{34)}$ and most likely forms the basis of frailty in patients with type 2 diabetes.

\section{PREVENTION AND MANAGEMENT OF FRAILTY AMONG DIABETIC PATIENTS}

Diabetes management may require assessments in the medical, psychological, functional, and social domains. In addition to the classic cardiovascular and microvascular disease, older adults with diabetes and frailty should be assessed for a group of conditions termed geriatric syndrome, which includes cognitive dysfunction, functional impairment, falls/ fractures, polypharmacy, depression, vision and hearing impairment, urinary incontinence, and nutritional problems. This comprehensive medical evaluation may provide a framework to determine targets and therapeutic approaches and to identify individuals with significantly impaired functional status such as visual and lower-extremity complications or cognitive impairment, which may impact their ability to self-manage their diabetes. $^{35)}$

Diabetes management strategies for robust older adults with diabetes are similar to those for younger adults. However, older adults with frailty should be provided individualized risk-minimization care plans according to the functional status and life expectancy. The American Geriatric Society suggests a glycosylated hemoglobin (HbAlc) target of 7.5\%-8.0\% for older adults. However, glycemic control targets may vary depending on the patient's frailty status. An HbAlc target of 7.0\%-7.5\% is suitable for functionally independent older adults with a reasonable life expectancy, while a target of $8 \%-9 \%$ is appropriate for older adults with frailty and those with dementia and a life expectancy of fewer than 10 years. ${ }^{36)}$

Sulfonylurea or insulin, which can increase the incidence of hypoglycemia, should be used with care in dependent patients with frailty and cognitive impairment. Glimepiride has a long half-life and can result in severe and prolonged hypoglycemia. If sulfonylureas are used, short-acting agents such as glipizide or gliclazide are preferred.

Metformin can be a first-line choice of treatment in older patients with diabetes and frailty. Patients with frailty should be monitored carefully for weight loss and gastrointestinal side effects. Thiazolidinedione should be used with care in patients with congestive heart failure and those at risk for falls or fractures. Dipeptidyl peptidase 4 (DPP-4) inhibitors are effective and reasonably safe in older adults. Because glucagon-like peptide 1-receptor agonists may be associated with gastrointestinal side effects and weight loss, they may not be desirable in underweight individuals with frailty. As only injectable forms are available, they should also be considered only when caregiver support is available. While sodium-glucose cotransporter 2 inhibitors appear to be well-tolerated by older adults, long-term data are scarce. ${ }^{35,36)}$ In addition, sodium-glucose transport protein 2 (SGLT2) inhibitors act as osmotic diuretics and are associated with weight loss as well as reduced blood pressure. Accordingly, these agents should be used with caution in older patients treated with antihypertensive agents, especially diuretics.

Diabetes nutritional therapy may have a protective effect against the development of frailty. Many older people lack adequate nutritional intake particularly that of protein, while also requiring increased dietary protein intake to compensate for age-related anabolic resistance. Older adults are recommended to consume 1.0 $1.2 \mathrm{~g}$ protein per kg of body weight per day to maintain and regain lean body mass and function. ${ }^{37)}$ Optimal nutrition with adequate protein intake combined with exercise programs, including aerobic and resistance training, could reduce the risks of sarcopenia and frailty. ${ }^{38-40)}$ Treatment of diabetic autonomic neuropathy in older adults is complex because of their poor tolerability to many pharmacologic treatment options. It is essential to search for the secondary causes and decrease the intake of medications that may contribute to the patient's symptoms. Lifestyle interventions such as diet alteration may help to reduce the need for pharmacologic treatments and their associated risks of adverse effects. ${ }^{41)}$ 


\section{CONCLUSION}

Diabetes is frequently accompanied by frailty and they share pathways leading to disability, morbidity, and mortality in older adults. Early recognition of frailty in older diabetic patients allows comprehensive multi-component interventions including physical exercise, nutritional support, and medication adjustment. Moreover, better strategies can be established for diabetes management, including setting glycemic goals, selecting anti-diabetic agents, and implementing other preventative interventions. Identification of the common mechanisms and the development of new therapeutic interventions are needed to meet the increasing demand for the management of older diabetic patients due to population aging.

\section{CONFLICT OF INTEREST DISCLOSURES}

The researchers claim no conflicts of interest.

\section{REFERENCES}

1. Kim DJ. The epidemiology of diabetes in Korea. Diabetes Metab J 2011;35:303-8.

2. Won JC, Lee JH, Kim JH, Kang ES, Won KC, Kim DJ, et al. Diabetes fact sheet in Korea, 2016: an appraisal of current status. Diabetes Metab J 2018;42:415-24.

3. Wong E, Backholer K, Gearon E, Harding J, Freak-Poli R, Stevenson $\mathrm{C}$, et al. Diabetes and risk of physical disability in adults: a systematic review and meta-analysis. Lancet Diabetes Endocrinol 2013;1:106-14.

4. Narayan KM, Boyle JP, Thompson TJ, Sorensen SW, Williamson DF. Lifetime risk for diabetes mellitus in the United States. JAMA 2003;290:1884-90.

5. Clegg A, Young J, Iliffe S, Rikkert MO, Rockwood K. Frailty in elderly people. Lancet 2013;381:752-62.

6. Fried LP, Tangen CM, Walston J, Newman AB, Hirsch C, Gottdiener J, et al. Frailty in older adults: evidence for a phenotype.J Gerontol A Biol Sci Med Sci 2001;56:M146-56.

7. Rockwood K, Mitnitski A. Frailty in relation to the accumulation of deficits. J Gerontol A Biol Sci Med Sci 2007;62:722-7.

8. Apostolo J, Cooke R, Bobrowicz-Campos E, Santana S, Marcucci M, Cano A, et al. Effectiveness of interventions to prevent pre-frailty and frailty progression in older adults: a systematic review. JBI Database System Rev Implement Rep 2018;16:140232.

9. Ottenbacher KJ, Graham JE, Al Snih S, Raji M, Samper-Ternent R, Ostir GV, et al. Mexican Americans and frailty: findings from the Hispanic established populations epidemiologic studies of the elderly. Am J Public Health 2009;99:673-9.

10. Cacciatore F, Testa G, Galizia G, Della-Morte D, Mazzella F, Langellotto A, et al. Clinical frailty and long-term mortality in elderly subjects with diabetes. Acta Diabetol 2013;50:251-60.

11. Hubbard RE, Andrew MK, Fallah N, Rockwood K. Comparison of the prognostic importance of diagnosed diabetes, co-morbidity and frailty in older people. Diabet Med 2010;27:603-6.

12. Jang HC. Sarcopenia, frailty, and diabetes in older adults. Diabetes Metab J 2016;40:182-9.

13. Saum KU, Dieffenbach AK, Muller H, Holleczek B, Hauer K, Brenner $\mathrm{H}$. Frailty prevalence and 10-year survival in community-dwelling older adults: results from the ESTHER cohort study. Eur J Epidemiol 2014;29:171-9.

14. Bouillon K, Kivimaki M, Hamer M, Shipley MJ, Akbaraly TN, Tabak A, et al. Diabetes risk factors, diabetes risk algorithms, and the prediction of future frailty: the Whitehall II prospective cohort study.J Am Med Dir Assoc 2013;14:851.

15. Chhetri JK, Zheng Z, Xu X, Ma C, Chan P. The prevalence and incidence of frailty in pre-diabetic and diabetic community-dwelling older population: results from Beijing Longitudinal Study of Aging II (BLSA-II). BMC Geriatr 2017; 17:47.

16. Kalyani RR, Corriere M, Ferrucci L. Age-related and disease-related muscle loss: the effect of diabetes, obesity, and other diseases. Lancet Diabetes Endocrinol 2014;2:819-29.

17. Krentz AJ, Viljoen A, Sinclair A. Insulin resistance: a risk marker for disease and disability in the older person. Diabet Med 2013; 30:535-48.

18. Park SW, Goodpaster BH, Strotmeyer ES, Kuller LH, Broudeau R, Kammerer C, et al. Accelerated loss of skeletal muscle strength in older adults with type 2 diabetes: the health, aging, and body composition study. Diabetes Care 2007;30:1507-12.

19. Morley JE, Malmstrom TK, Rodriguez-Manas L, Sinclair AJ. Frailty, sarcopenia and diabetes. J Am Med Dir Assoc 2014;15:853-9.

20. Lee S, Lee S, Harada K, Bae S, Makizako H, Doi T, et al. Relationship between chronic kidney disease with diabetes or hypertension and frailty in community-dwelling Japanese older adults. Geriatr Gerontol Int 2017;17:1527-33.

21. Goulet ED, Hassaine A, Dionne IJ, Gaudreau P, Khalil A, Fulop $\mathrm{T}$, et al. Frailty in the elderly is associated with insulin resistance of glucose metabolism in the postabsorptive state only in the presence of increased abdominal fat. Exp Gerontol 2009;44: 740-4.

22. Castro-Rodriguez M, Carnicero JA, Garcia-Garcia FJ, Walter S, Morley JE, Rodriguez-Artalejo F, et al. Frailty as a major factor in the increased risk of death and disability in older people with diabetes. J Am Med Dir Assoc 2016;17:949-55. 
23. Liccini A, Malmstrom TK. Frailty and sarcopenia as predictors of adverse health outcomes in persons with diabetes mellitus. J Am Med Dir Assoc 2016;17:846-51.

24. Sinclair A, Dunning T, Rodriguez-Manas L. Diabetes in older people: new insights and remaining challenges. Lancet Diabetes Endocrinol 2015;3:275-85.

25. Abdelhafiz AH, Rodriguez-Manas L, Morley JE, Sinclair AJ. Hypoglycemia in older people: a less well recognized risk factor for frailty. Aging Dis 2015;6:156-67.

26. Tilling LM, Darawil K, Britton M. Falls as a complication of diabetes mellitus in older people. J Diabetes Complications 2006;20:158-62.

27. Huang ES, Karter AJ, Danielson KK, Warton EM, Ahmed AT. The association between the number of prescription medications and incident falls in a multi-ethnic population of adult type2 diabetes patients: the diabetes and aging study. J Gen Intern Med 2010;25:141-6.

28. Tamura Y, Ishikawa J, Fujiwara Y, Tanaka M, Kanazawa N, Chiba $\mathrm{Y}$, et al. Prevalence of frailty, cognitive impairment, and sarcopenia in outpatients with cardiometabolic disease in a frailty clinic. BMC Geriatr 2018;18:264.

29. Munshi MN. Cognitive dysfunction in older adults with diabetes: what a clinician needs to know. Diabetes Care 2017;40:461-7.

30. Feinkohl I, Aung PP, Keller M, Robertson CM, Morling JR, McLachlan S, et al. Severe hypoglycemia and cognitive decline in older people with type 2 diabetes: the Edinburgh type 2 diabetes study. Diabetes Care 2014;37:507-15.

31.Sakkas GK, Kent-Braun JA, Doyle JW, Shubert T, Gordon P, Johansen KL. Effect of diabetes mellitus on muscle size and strength in patients receiving dialysis therapy. Am J Kidney Dis 2006;47:862-9.

32. Pupim LB, Heimburger O, Qureshi AR, Ikizler TA, Stenvinkel P. Accelerated lean body mass loss in incident chronic dialysis patients with diabetes mellitus. Kidney Int 2005;68:2368-74.

33. Morley JE, Baumgartner RN, Roubenoff R, Mayer J, Nair KS. Sarcopenia.J Lab Clin Med 2001;137:231-43.
34. Janssen I, Heymsfield SB, Ross R. Low relative skeletal muscle mass (sarcopenia) in older persons is associated with functional impairment and physical disability. J Am Geriatr Soc 2002;50: 889-96.

35. American Diabetes Association. 12. Older adults: standards of medical care in diabetes. 2019. Diabetes Care 2019;42(Suppl 1):S139-47.

36. American Geriatrics Society Expert Panel on Care of Older Adults with Diabetes Mellitus, Moreno G, Mangione CM, Kimbro L, Vaisberg E. Guidelines abstracted from the American Geriatrics Society guidelines for improving the care of older adults with diabetes mellitus: 2013 update. J Am Geriatr Soc 2013;61:2020-6.

37. Bauer J, Biolo G, Cederholm T, Cesari M, Cruz-Jentoft AJ, Morley JE, et al. Evidence-based recommendations for optimal dietary protein intake in older people: a position paper from the PROT-AGE Study Group. J Am Med Dir Assoc 2013;14:54259.

38. Sinclair AJ, Abdelhafiz A, Dunning T, Izquierdo M, Rodriguez Manas L, Bourdel-Marchasson I, et al. An international position statement on the management of frailty in diabetes mellitus: summary of recommendations 2017. J Frailty Aging 2018;7:1020.

39. Tieland M, Dirks ML, van der Zwaluw N, Verdijk LB, van de Rest O, de Groot LC, et al. Protein supplementation increases muscle mass gain during prolonged resistance-type exercise training in frail elderly people: a randomized, double-blind, placebo-controlled trial. J Am Med Dir Assoc 2012;13:713-9.

40. Rahi B, Morais JA, Dionne IJ, Gaudreau P, Payette H, Shatenstein B. The combined effects of diet quality and physical activity on maintenance of muscle strength among diabetic older adults from the NuAge cohort. Exp Gerontol 2014;49:40-6.

41. Scheinberg N, Salbu RL, Goswami G, Cohen K. Treatment of diabetic autonomic neuropathy in older adults with diabetes mellitus. Consult Pharm 2016;31:633-45. 